Discussion Conclusions on whether condom use wanes among dual users may depend on the composition of groups compared. Future investigation of condom use among $\mathrm{HC}$ users should address this potential source of bias. If interpreted alongside each sub-groups' risk patterns for STD and unplanned pregnancy, the correlates of dual use can inform dual use interventions.

\section{P2-S1.09 ASSOCIATIONS BETWEEN EARLY SEXUAL DEBUT AND TWO TYPES OF SEXUAL RISK BEHAVIOUR IN NOVA SCOTIA ADOLESCENTS}

doi:10.1136/sextrans-2011-050108.287

D Langille, P Andreou, K Wilson. Dalhousie University, Halifax, Canada

Background This study sought to test associations between early sexual debut (first vaginal intercourse before age 15) and later sexual risk-taking among adolescents aged 15 to 19

Methods Self-report surveys were administered to 433 high school students in Yarmouth, Nova Scotia, Canada in May 2009. The surveys asked about general demographics, socioeconomic status, substance use, depression, peers' attitudes towards sex, and the sexual behaviours of students. Categorical principal components analysis was used to determine whether several of the outcome variables could be combined, and logistic regressions were used to assess the associations between predictor and outcome variables.

Results Two factors emerged among the risk behaviours: i) recreational sex (having many sexual partners, having sex while using substances and having casual partners); and; ii) inconsistent condom use, each of which was predicted by a distinct set of variables. Adjusted logistic regressions revealed that early sexual debut was associated with an increased risk of having used condoms inconsistently in the last year (OR 4.7; 95\% CI 1.0 to 21.5) and having had recreational sex in the same period (OR 2.5; 95\% CI 0.1 .0 to 6.4) among girls. The association between early debut and sexual risk behaviours was not seen in boys.

Conclusions Early sexual debut predicted of sexual risk taking among girls but not boys. Our findings offer a partial replication and expansion on recent research examining this issue. Asking high school students about age of their sexual debut and other factors might allow healthcare professionals to identify high risk individuals.

\section{P2-S1.10 IDENTIFYING KEY ELEMENTS DESCRIBING SEXUAL BEHAVIOUR IN THE DANISH POPULATION: A QUALITATIVE STUDY}

doi:10.1136/sextrans-2011-050108.288

${ }^{1} \mathrm{M} J$ Jørgensen, ${ }^{2} \mathrm{H}$ T Maindal, ${ }^{2} \mathrm{~F}$ Olesen, ${ }^{1} \mathrm{~B}$ Andersen. ${ }^{1}$ Randers Regional Hospital, Randers NØ, Denmark; ${ }^{2}$ Aarhus University, Aarhus C, Denmark

Background Surveying the sexual behaviour of the general population serves to identify key points of intervention, monitor the effect of such an intervention and interpret changes in the spread of sexually transmitted infections over time. Validated questionnaires describing sexual behaviour could be adapted from other countries, but due to cultural differences this could result in the need to add additional or other questions in order to describe the important Danish issues sufficiently. With a view to designing and initiating a sexual behaviour surveillance program in Denmark, the aim of this qualitative study was to identify points of particular importance to adolescents' sexual behaviour.
Method We conducted four semi-structured focus group interviews with a total of 19 sexually experienced adolescents aged 18 to 23 . Boys and girls were interviewed separately. Each group contained pupils from the same Danish Folk High School, but with different social and educational backgrounds. The interview guide was developed on the basis of literature reviews and hypotheses based on many years of experience with sexually transmitted infections from an epidemiological perspective as well as treatment of patients with sexually transmitted infections. Data were transcribed verbatim and analysed using qualitative description.

Results We identified four major categories of risk behaviour: 1) Alcohol consumption is associated with "no condom use". 2) Nights on the town and meetings in foreign counties or at festivals are associated with one night stands and often lead to unsafe sex. 3) Low self-esteem increases the risk of pushing one's personal boundaries, thus resulting in promiscuous sexual behaviour. 4) Increased sexual experience is associated with lack of condom use. Surprisingly, the informants did not consider drug abuse and internet dating to be triggers of unsafe sex see Abstract P2-S1.10 Figure 1.

Conclusion Danish adolescents identified four key elements that could lead to unsafe sex. These results differed slightly from our expectations and will be included in a sexual behaviour questionnaire to describe important elements influencing the sexual behaviour of Adolescents.

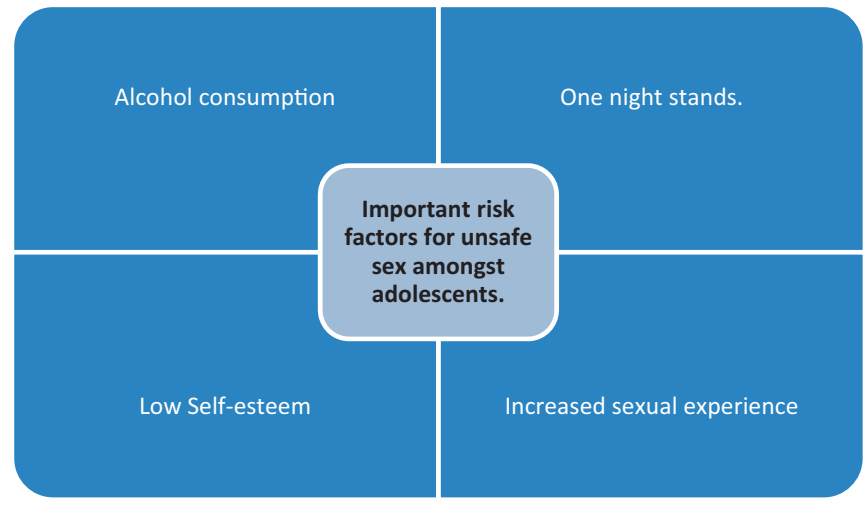

Abstract P2-S1.10 Figure 1 Important risk factors for unsafe sex among adolescents.

\section{P2-S1.11 FACTORS SURROUNDING LACK OF CONDOM USE AT FIRST INTERCOURSE AND LATER ONSET OF CONDOM USE: A STUDY OF COLLEGE-AGE MEN}

doi:10.1136/sextrans-2011-050108.289

${ }^{1} \mathrm{~L}$ Warner, ${ }^{1} \mathrm{~K} \mathrm{M},{ }^{1} \mathrm{~L}$ Gavin, ${ }^{2} \mathrm{M}$ Macaluso. ${ }^{1} \mathrm{CDC}$, Atlanta, USA; ${ }^{2}$ Cincinatti childrens center, Cincinatti, USA

Background Condom use at first coitus is associated with greater lifetime condom use. Little is known, however, about factors which influence non-use of condoms at first coitus.

Methods Data were analysed from a cross-sectional study on condom use behaviours conducted among 98 heterosexual male students attending two Georgia universities. Men were asked to recall condom use during first and subsequent coitus. Multivariable logistic regression was conducted to evaluate factors associated with non-use of condoms at first coitus and how non-use influenced their future condom beliefs and use.

Results Participant averaged 22.4 years at interview, 16.8 years at first intercourse (range: $13-23$ ), and 5.6 years (range:0-12) between first condom use and time of interview. Overall, 47 men (48\%) reported not using condoms at first coitus, and not initiating use until an average of 13 acts after sexual debut (med=5; range:1-100). 
Reasons for eventual condom initiation included: concern about infection/pregnancy (53\%), partner insistence (32\%), and condoms being available (15\%). Compared with men who used condoms at first intercourse, men initiating use afterwards were significantly more likely to report their first condom experience was negative (62\% vs $35 \%, \mathrm{aOR}=2.8,95 \%$ CI 1.1 to 7.2 ), and were less inclined to use condoms subsequently based on this first experience (34\% vs $14 \%, \mathrm{aOR}=3.4,95 \%$ CI 1.2 to 8.8). However, measurement of condom use during most recent coitus did not differ significantly between men who initiated condom use after vs at their first intercourse both at last sex (43\% vs $55 \%$ ) and for both consistent use during the last month (43\% vs $39 \%$ ) and year (26\% vs $20 \%$ ).

Conclusions Public health efforts should emphasise the importance of condom use at first intercourse. Counselling should be provided to help prepare men for a positive first experience with condoms, given that this experience may affect immediate subsequent condom use following sexual debut.

\section{P2-S1.12 PROJECT PREPARE TANZANIA: ENGAGING MULTIPLE STAKEHOLDERS TO DEVELOP A SCHOOL-BASED SEXUAL HEALTH CURRICULUM FOR ADOLESCENTS}

doi:10.1136/sextrans-2011-050108.290

${ }^{1} \mathrm{R}$ McCree-Hale, ${ }^{2} \mathrm{~L}$ Kajula-Maonga, ${ }^{2}$ Tatizo Ramatu Sheya wa Mnyakaya, ${ }^{2} \mathrm{~J}$ Joachim, ${ }^{2} \mathrm{~K}$ Mrumbi, ${ }^{2} \mathrm{E}$ John, ${ }^{2} \mathrm{~S}$ Kaaya. ${ }^{1}$ Management and Development for Health, Dar es Salaam, United Republic of Tanzania; ${ }^{2}$ Muhimbili University, Dar es Salaam, United Republic of Tanzania

Background The objective of this formative research was to engage multiple stakeholders to identify specific factors that may influence program development for Project Prepare Tanzania- an innovative, school-based sexual health curriculum for young adolescents in Tanzania.

Methods Focus group discussions (FGDs) and structured interviews were used to elicit data for program development from students, teachers, parents, and healthcare workers. 12 FGDs were conducted with male and female adolescents $(n=90)$ from 6 randomly selected schools in Dar es Salaam, Tanzania. Individual interviews $(n=16)$ were conducted with boys $(n=8)$ and girls $(n=8)$. Key informant interviews were conducted with teachers $(n=12)$ and parents $(\mathrm{n}=12)$. Community social workers $(\mathrm{n}=6)$ and healthcare workers $(n=4)$ also participated in key informant interviews. Interview and FGD guides were designed to elicit data on: sexuality communication, self-efficacy, parenting and sexuality, inclusion of sexual health education in schools and knowledge, attitudes, and perceptions related to peer-education and skills-building for sexual health. NVivo 8 software was used to analyse data and help develop salient qualitative themes. Results Salient themes were related to sexuality communication, attitudes towards skills building for condom use and negotiation of safe sex, access to correct information for sexual health, and partnership building to strengthen links between stakeholders. Factors that may influence communication about sexual health among adolescents include: fear associated with talking to parents, fear of being perceived as immoral, and inadequate opportunities to discuss sexuality including puberty. Parents and teachers reported needing skills to discuss puberty, sexually transmitted infections and sexual health. Teachers indicated that curricula should be expanded to include a sexual health component. There is a need for values clarification between parents and teachers regarding teaching about condoms and healthy sexual scripts for negotiation of safe sex. All participants suggested strengthening links between stakeholders to promote more communication.

Conclusions Project Prepare Tanzania will address the facilitators and barriers identified in this formative research to develop a culturally acceptable, innovative and sustainable sexual health curriculum for adolescents.

\section{P2-S1.13 WHY INDIVIDUALS DO NOT RETURN THEIR REQUESTED CHLAMYDIA TRACHOMATIS (CT) HOME COLLECTION KIT: RESULTS FROM A QUALITATIVE STUDY}

doi:10.1136/sextrans-2011-050108.291

R KoekenbierL Kalma, F Zuure, U Davidovich; On behalf of the Chlamydia Screening Implementation CSI project group. Amsterdam Public Health Service Amsterdam, Netherlands

Background A systematic and selective screening programme for CT was set up in the Netherlands, in which adolescents were invited by a personal letter to request a home collection kit online (http:// www.chlamydiatest.nl). After collection, the kit could be returned to the laboratory by mail. In the first screening round (2008/2009), $20 \%(52346 / 261053)$ of the adolescents requested a kit. Of those $22 \%$ (11317/52 346) did not return the kit (non-testers). Here, we describe a qualitative study examining motives of non-testers for requesting a home collection kit, and their barriers for using it.

Methods Semistructured telephone interviews were conducted with non-testers from the second screening round (2009/2010) until data saturation was reached $(n=25)$. They were invited for participation by email. Transcribed interviews were analysed qualitatively using Flexible Content Analysis, and interpreted using health behavioural theories that is, the Health Action Process Approach (HAPA).

Results Motives of participants to request a kit related to perceived risk for CT were: for certainty/reassurance, having complaints, or to take responsibility for one's own health. Motives related to specific features of the screening procedures were: it avoids invasive screening at STI-clinic or GP, procedures are simple, or because the screening is anonymous. Barriers for using a kit related to perceived risk for CT were: recently being tested, worries about the consequences of a positive test-result on the relationship, having no time, giving testing no priority, or laziness. Barriers related to specific features of the screening were: making wrong assumptions about the term of preservability of an unused test kit or about the term of the screening round, the user manual is unclear, being afraid to make mistakes during the collection procedures, the method of collection is unpleasant, or worries about privacy.

Conclusions Motives to request the CT home collection kit were related to the participants perceived risk of acquiring CT, and to advantages of the screening procedures compared to conventional testing. Barriers for using the test kit were related to low personal risk perception, unclear procedures of the screening, and fear for a positive test result. To overcome these barriers, the ease of contracting CT, and consequences of an untreated infection, should be emphasised. Furthermore, screening procedures should be optimised according to the users experiences.

\section{P2-S1.14 THE ACCURACY OF PERCEPTIONS ABOUT SEXUAL CONCURRENCY AMONG PREGNANT ADOLESCENTS AND THEIR PARTNERS AND THE INFLUENCE OF SELF- REPORTED CONCURRENCY}

doi:10.1136/sextrans-2011-050108.292

${ }^{1} \mathrm{~A}$ Swartzendruber, ${ }^{2} \mathrm{~L}$ Niccolai, ${ }^{3} \mathrm{~J}$ Zenilman, ${ }^{3} \mathrm{~J}$ Jennings, ${ }^{2} \mathrm{H}$ Sipsma, ${ }^{2} \mathrm{~A}$ Arnold, ${ }^{2}$ T Kershaw. ' Johns Hopkins Bloomberg School of Public Health, Baltimore, USA; ${ }^{2}$ Yale School of Public Health, USA; 'Johns Hopkins University School of Medicine, USA

Background Inaccurate perceptions about sexual partner concurrency are associated with current STI status. While high STI rates are reported among pregnant adolescents, studies have not investigated the accuracy of perceptions about sexual concurrency among young expecting couples nor explored factors related to inaccurate perceptions. Without open communication, individuals may inaccurately assume their partner's behaviour is like their own. The 